\title{
Possible therapeutic impact of the iron chelation on renal fibrosis
}

\author{
Kouichi Tamura, Kazushi Uneda, Kengo Azushima, Hiromichi Wakui and Kotaro Haruhara
}

Hypertension Research (2015) 38, 455-456; doi:10.1038/hr.2015.60; published online 16 April 2015

$\mathrm{C}^{\mathrm{h}}$ hronic kidney disease (CKD) patients are reportedly increasing in number worldwide and exhibit a significantly enhanced risk of cardiovascular complications. In addition, these patients also have an increased risk of CKD progression to end-stage renal disease, which requires hemodialysis or renal transplantation therapy. ${ }^{1}$ There is increasing evidence of interorgan cross talk mediated by a variety of factors that may accelerate pathologic processes and the progression of organ dysfunction in the renal and cardiovascular systems. $^{2}$ In the kidney, the process of tubulointerstitial fibrosis is characterized by extracellular matrix deposition, interstitial myofibroblast proliferation and the infiltration of inflammatory mononuclear cells, which are thought to have an important role in the pathogenesis of CKD. ${ }^{3}$ Therefore, amelioration of renal interstitial fibrosis is important to halt the progression of CKD.

Although iron is an elementary trace metal that is essential for viability in most organisms, excess iron can lead to Fenton/HaberWeiss catalytic reactions and the generation of free radicals that are damaging to lipid membranes, proteins and nucleic acids, and cause organ damage. Thus, to maintain normal physiological tissue function, it is critically important to control the levels of circulating iron and tissue iron stores by sophisticated functional modulation of iron transporters and iron-binding proteins. ${ }^{4}$

K Tamura, K Uneda, K Azushima, $H$ Wakui and $K$ Haruhara are at Department of Medical Science and Cardiorenal Medicine Yokohama City University Graduate School of Medicine, Yokohama, Japan; $\mathrm{K}$ Haruhara is at Division of Nephrology and Hypertension Department of Internal Medicine, The Jikei University School of Medicine, Tokyo, Japan

E-mail: tamukou@med.yokohama-cu.ac.jp
Naito et al. ${ }^{5}$ are continuously investigating putative roles of iron in the pathogenesis of chronic kidney disease (CKD) by employing a rat model of CKD that uses 5/6 nephrectomy in Sprague-Dawley rats, which exhibit hypertension, glomerulosclerosis and renal interstitial fibrosis. Naito et al. ${ }^{5}$ previously reported that renal iron accumulation and the expression of intracellular iron transport proteins were increased in the tubules of this CKD model. These authors also examined the effect of dietary iron restriction on renal damage and mineralocorticoid receptor signaling in this model and showed that dietary iron restriction ameliorated the development of renal damage and hypertension by inhibiting renal mineralocorticoid receptor signaling. In addition, Naito et al. ${ }^{5}$ investigated the therapeutic effects of iron restriction on pre-existing hypertension and renal damage in the same rat model of CKD and showed that iron restriction prevented further deterioration of pre-existing renal damage through a similar inhibition of renal mineralocorticoid receptor signaling. ${ }^{6}$ They also show that dietary iron restriction attenuated the development of hypertension and renal injury in aldosterone/salt-induced hypertensive mice. ${ }^{7}$

In this issue, Naito et al. further extend their above-mentioned studies and examine the effects of an oral iron chelator, deferasirox (DFX), on renal fibrotic lesion development in the $5 / 6$ nephrectomy rat model of CKD. ${ }^{8}$ In contrast to the inhibitory effect of dietary iron restriction on the development of hypertension and glomerulosclerosis in the CKD rat model, iron chelation by DFX did not affect hypertension or glomerulosclerosis. However, iron chelation by DFX did suppress fibrotic and inflammatory responses in the rats despite DFX treatment having no obvious effects on BP or the development of glomerular lesions. ${ }^{8}$

This is an important finding because renal interstitial fibrosis increases nocturnal BP, which contributes to a pathological diurnal BP rhythm and promotes renal deterioration in CKD patients. ${ }^{9}$ In addition, renal interstitial fibrosis is a common feature of various types of end-stage renal disease. DFX treatment inhibited the upregulation of the renal expression of lipocalin 2, an important stimulator of renal fibrosis. ${ }^{8}$ In addition, the transforming growth factor $\beta$ (TGF- $\beta$ ) pathway (TGF- $\beta$ and Smad3) is known to be involved in the pathogenesis of fibrotic lesions in the kidney, ${ }^{10}$ and the oxidativecarbonyl stress pathway, including nicotinamide adenine dinucleotide phosphate oxidase that is suggested to be involved in the pathophysiology of renal fibrosis as well as the development of hypertension. ${ }^{11,12}$ In this issue, Naito et al. demonstrated that iron chelation by DFX inhibits these fibrosis-, oxidative stress- and inflammation-related genetic changes in the kidney. ${ }^{8}$

Collectively, the authors suggest that iron chelation may be a therapeutic strategy for the inhibition of renal fibrosis in CKD. However, iron chelation with DFX did not affect proteinuria, glomerulosclerosis or podocyte injury associated with decreased renal intestinal fibrosis in $\mathrm{CKD}$ rats in the present study. ${ }^{8}$ Therefore, as described by the authors, the attenuation of renal interstitial fibrosis by iron chelation may be a secondary effect that does not contribute to the mitigation of renal damage itself. Nevertheless, the results are interesting for understanding the pathophysiology of renal fibrosis in CKD. Recently, Ikeda et al. ${ }^{13}$ showed that iron chelation by DFX prevents renal tubulointerstitial fibrosis in mice with surgically induced 


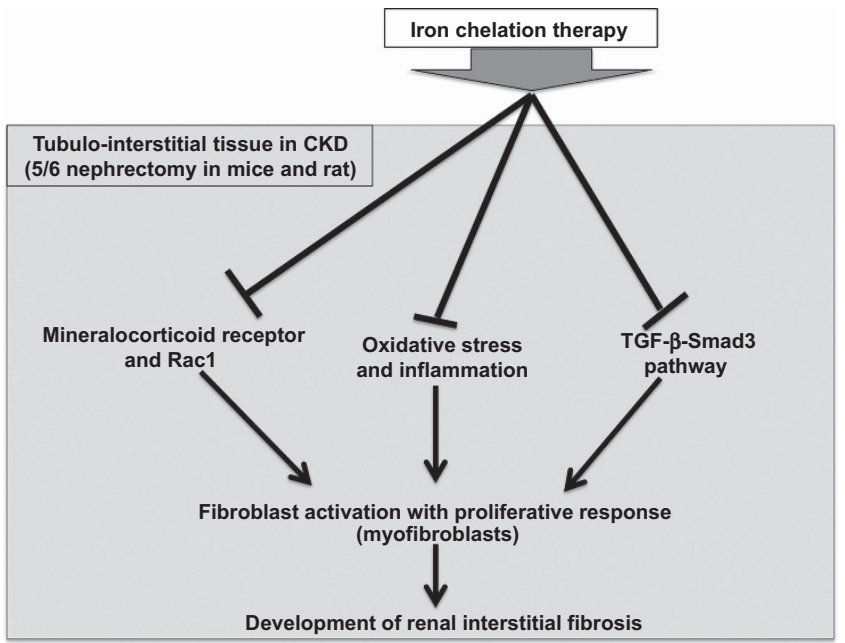

Figure 1 Schematic representation of the possible mechanism by which iron chelation ameliorates renal interstitial fibrosis in the 5/6 nephrectomy CKD (chronic kidney disease) model.

unilateral ureter obstruction by regulating TGF- $\beta$-Smad signaling, oxidative stress and inflammatory responses. Therefore, iron chelation is emerging as a possible target of interest for the efficient treatment of renal interstitial fibrosis (Figure 1). Further experimental and clinical evidence are needed to determine the exact effects of iron chelation. ${ }^{14}$

\section{CONFLICT OF INTEREST}

The authors declare no conflict of interest.

\section{ACKNOWLEDGEMENTS}

KT received a research grant or honoraria from Takeda, Daiichi-Sankyo, Kyowa-hakko Kirin, Shionogi, Dainippon-Sumitomo, Novartis, Chu-gai, Mochida, MSD, Tanabe Mitsubishi,
Boehringer Ingelheim, Astellas, Pfizer, AstraZeneca and Sanofi.

1 Hallan SI, Matsushita K, Sang Y, Mahmoodi BK, Black C, Ishani A, Kleefstra N, Naimark D, Roderick P, Tonelli M, Wetzels JF, Astor BC Gansevoort RT, Levin A, Wen CP, Coresh J. Age and association of kidney measures with mortality and end stage renal disease. JAMA 2012; 308: 2349-2360.

2 Charytan DM, Fishbane S, Malyszko J, McCullough PA Goldsmith D. Cardiorenal syndrome and the role of the bone-mineral axis and anemia. Am J Kidney Dis 2015 (e-pub ahead of print 26 February 2015).

3 Iwano M, Neilson EG. Mechanisms of tubulointerstitial fibrosis. Curr Opin Nephrol Hypertens 2004; 13 279-284.

4 Cairo G, Recalcati S, Pietrangelo A, Minotti G. The iron regulatory proteins: targets and modulators of free radical reactions and oxidative damage. Free Radic Biol Med 2002: 32: 1237-1243.
5 Naito Y, Fujii A, Sawada H, Hirotani S, Iwasaku T, Eguchi A, Ohyanagi M, Tsujino T, Masuyama T. Effect of iron restriction on renal damage and mineralocorticoid receptor signaling in a rat model of chronic kidney disease. J Hypertens 2012; 30 2192-2201.

6 Naito Y, Fujii A, Sawada H, Hirotani S, Iwasaku T, Okuhara $Y$, Eguchi A, Ohyanagi M, Tsujino $T$, Masuyama T. Dietary iron restriction prevents further deterioration of renal damage in a chronic kidney disease rat model. J Hypertens 2013; 31: 1203-1213.

7 Sawada H, Naito $Y$, Oboshi M, Iwasaku T, Okuhara $Y$, Morisawa D, Eguchi A, Hirotani S, Masuyama T. Iron restriction inhibits renal injury in aldosterone/salt induced hypertensive mice. Hypertens Res 2015; 38 317-322.

8 Naito Y, Fujii A, Sawada H, Oboshi M, Iwasaku T, Okuhara Y, Morisawa D, Eguchi A, Hirotani S, Masuyama T. Association between renal iron accumulation and renal interstitial fibrosis in a rat model of chronic kidney disease. Hypertens Res 2015; 38 463-470.

9 Haruhara K, Tsuboi N, Koike K, Fukui A, Miyazaki Y, Kawamura T, Ogura M, Yokoo T. Renal histopathological findings in relation to ambulatory blood pressure in chronic kidney disease patients. Hypertens Res 2015; 38: 116-122.

10 Chevalier RL, Forbes MS, Thornhill BA. Uretera obstruction as a model of renal interstitial fibrosis and obstructive nephropathy. Kidney Int 2009; 75 : 1145-1152.

11 Chen X, Mori T, Guo Q, Hu C, Ohsaki Y, Yoneki Y, Zhu W, Jiang $Y$, Endo S, Nakayama K, Ogawa S, Nakayama M, Miyata T, Ito S. Carbonyl stress induces hypertension and cardio-renal vascular injury in Dahl salt-sensitive rats. Hypertens Res 2013; 36 361-367.

12 Sundaram A, Siew Keah L, Sirajudeen KN, Singh HJ. Upregulation of catalase and downregulation of glutathione peroxidase activity in the kidney precede the development of hypertension in prehypertensive SHR. Hypertens Res 2013; 36: 213-218.

13 Ikeda Y, Ozono I, Tajima S, Imao M, Horinouchi $Y$ Izawa-Ishizawa Y, Kihira Y, Miyamoto L, Ishizawa K, Tsuchiya K, Tamaki T. Iron chelation by deferoxamine prevents renal interstitial fibrosis in mice with unilateral ureteral obstruction. PLOS One 2014, 9: e89355.

14 Mack M, Yanagita M. Origin of myofibroblasts and cellular events triggering fibrosis. Kidney Int 2015; 87: 297-307. 